LMU-TPW 92-31

January 1993

\title{
ON THE GEOMETRICAL STRUCTURE OF COVARIANT ANOMALIES IN YANG-MILLS THEORY
}

\author{
Gerald Kelnhofer* \\ Sektion Physik der Ludwig-Maximilians-Universität \\ Theresienstr. 37, 8000 München 2 \\ Germany
}

\begin{abstract}
Covariant anomalies are studied in terms of the theory of secondary characteristic classes of the universal bundle of Yang-Mills theory. A new set of descent equations is derived which contains the covariant current anomaly and the covariant Schwinger term. The counterterms relating consistent and covariant anomalies are determined. A geometrical realization of the BRS/anti-BRS algebra is presented which is used to understand the relationship between covariant anomalies in different approaches.
\end{abstract}

*)Erwin Schrödinger fellow, supported by "Fonds zur Förderung der wissenschaftlichen Forschung 


\section{INTRODUCTION}

There has been much progress in investigating the phenomenon of anomalies in quantum field theory during the last years. Anomalies manifest themselves in two different ways, known as consistent and covariant anomalies. Cohomological methods have been proved to be useful for analyzing the mathematical structure of the consistent anomaly. Its algebraic origin can be traced back to the Weil algebra structure of the BRS algebra [DTV]. The BRS algebra is based on the multiplet $\left(A, c, F_{A}, \delta, d_{P}\right)$, where $A$ is a connection with curvature $F_{A}$ in a principal $G$-bundle $P$ over $n$-dimensional space-time $M, c$ is the (Faddeev-Popov) ghost, $\delta$ is the BRS operator and $d_{P}$ is the exterior derivative on $P$. The consistent anomalies are related to the BRS cohomology $\delta$ modulo $d_{P}$ and are generated by the consistent descent equations [S, Zu].

The universal bundle construction of Atiyah and Singer [AS] provides a geometrical framework to realize the BRS multiplet and the corresponding BRS transformations. Application of the theory of secondary characteristic forms [C] finally leads to the consistent descent equations.

There have been various attempts to understand covariant anomalies from a cohomological viewpoint [Ts, Z, AAG, K1]. An algebraic method has been suggested in [Ts] and [Z] to derive covariant anomalies such as the covariant current anomaly [BZ] and the covariant commutator anomaly (Schwinger term) [NT, K2] by transgression from a Chern-Simons form. The basic idea is to enlarge the usual BRS algebra by introducing a new field $\bar{c}$, the anti-ghost, together with a nilpotent operator $\bar{\delta}$, which is called anti-BRS operator. This BRS/anti-BRS algebra provides an algebraic framework to formulate an integrability condition for covariant anomalous terms of arbitrary ghost degree. In [Ts, Z], a solution of this covariance condition was obtained by introducing certain homotopy operators. A geometrical description of the BRS/anti-BRS algebra has been proposed in [Z]. But this attempt is not satisfactory since the global meaning of the anti-ghost, being defined as part of a connection in the universal bundle, and of the anti-BRS operator is not very obvious.

A different characterization of the covariant anomaly has been already suggested by Bonora and Cotta-Ramusino [BC1]. Their considerations are based on a reformulation of the anomaly problem in the total space of the given principal fibre bundle $P$. They found that the non-integrated covariant current anomaly belongs to a certain class in the $n$th local de-Rham cohomology group of ghost degree one built with cocycles of the total space $P$ linear in the ghost. One aim of this paper is to understand the relationship between these different approaches.

We present a geometrical approach to the calculation of covariant anomalies in terms of secondary characteristic classes of the universal bundle. Thereby a new set of descent equations is derived which contains the covariant current anomaly and the covariant Schwinger term. The result of [BC1] follows directly from our analysis.

In order to relate the different approaches we shall construct a geometrical realization of the BRS/anti-BRS algebra on an appropriate $G$ bundle. Ghost and anti-ghost turn out to be components of a canonical connection in this bundle. Our formalism naturally extends to this generalized bundle. Finally we obtain a system of descent equations whose solutions satisfy the covariance condition. The 
possibility to characterize covariant anomalies in terms of local cohomology turns out to be a consequence of the proposed formalism.

This paper is organized as follows: In Sec. 2, we fix some notations for the geometry of Yang-Mills fields. Then we review the universal bundle construction of Atiyah and Singer and derive the consistent descent equations. In Sec. 3 we present our geometrical approach and derive the covariant descent equations. The relation between consistent and covariant anomalies is investigated. Then we compare our formalism with the results of [BC1]. An appropriate geometrical realization of the BRS/anti-BRS multiplet is constructed in Sec. 4. Then we discuss the relationship between the covariant anomaly in the two different approaches.

\section{The COnsistent Descent EQUations}

In this section we shall review the geometrical structure of consistent anomalies based on the universal bundle construction [AS]. In order to fix notation we shall begin with a short review of the geometry of Yang-Mills fields.

Let $(M, g)$ be a $n$-dimensional, compact, oriented Riemannian manifold, $\pi_{P}: P \rightarrow$ $M$ a principal $G$-bundle over $M$, where the structure group $G$ is a compact Lie group and whose Lie algebra will be denoted by $\mathfrak{g}$. The principal right action is $(p, g) \mapsto p g$. The space of (irreducible) connections on $P$ will be denoted by $\mathcal{A}$ and the gauge group $\mathcal{G}$ is identified with the group of based bundle automorphisms (modulo the centre of $G$ ) of $P$. So $\pi_{\mathcal{A}}: \mathcal{A} \rightarrow \mathcal{M}$ becomes a principal- $\mathcal{G}$ bundle over the gauge orbit space $\mathcal{M}$ with principal action $\mathcal{R}^{\mathcal{A}}(A, u):=u^{*} A[\mathrm{MV}]$. Every $u \in \mathcal{G}$ defines a section $\hat{u}$ of the associated bundle $P \times_{G} G$, given by $u(p)=p \hat{u}(p)$. Associated to $P$ by the adjoint action of $G$ in $\mathfrak{g}$ is the bundle $a d P:=P \times_{G} \mathfrak{g}$. The bundles $\wedge^{q} T^{*} M \otimes a d P$ have canonical metrics $($,$) induced by the metric g$ and the Killing form on $\mathfrak{g}$ and thus the space of adP-valued $q$-forms

$$
\Omega^{q}(M, a d P):=\Gamma\left(\wedge^{q} T^{*} M \otimes a d P\right)
$$

has an inner product, given by

$$
<\phi_{1}, \phi_{2}>:=\int_{M}\left(\phi_{1}, \phi_{2}\right) .
$$

Every $A \in \mathcal{A}$ defines a covariant exterior derivative $d_{A}: \Omega^{q}(M$, adP $) \rightarrow \Omega^{q+1}(M$, adP $)$. Its adjoint will be denoted by $d_{A}^{*}$. The Lie algebra $L i e \mathcal{G}$ of $\mathcal{G}$ can be identified with $\Omega^{0}(M, a d P)$ and the fundamental vector field $Z^{\mathcal{A}}$ with respect to $\mathcal{R}^{\mathcal{A}}$ is given by $Z_{\xi}^{\mathcal{A}}(A)=d_{A} \xi$, where $\xi \in \Omega^{0}(M, a d P)$. Since $\mathcal{A}$ is an affine space the tangent bundle is $T \mathcal{A}=\mathcal{A} \times \Omega^{1}(M, a d P)$. Note that $\Omega^{q}(M, a d P)$ is isomorphic with the space of ad-equivariant, horizontal $\mathfrak{g}$-valued $q$-forms on $P$, denoted by $\Omega_{e q, h}^{q}(P, \mathfrak{g})$.

There exists a canonical connection $\alpha$ on the bundle $\mathcal{A} \rightarrow \mathcal{M}$, given by

$$
\alpha_{A}\left(\tau_{A}\right)=\left(d_{A}^{*} d_{A}\right)^{-1} d_{A}^{*}\left(\tau_{A}\right)
$$

Thus every tangent vector $\tau_{A} \in T_{A} \mathcal{A}$ can be split into a horizontal and vertical part, written as

$$
\tau_{A}=\tau_{A}^{h}+\tau_{A}^{\mathrm{ver}}:=\left(1-d_{A} \alpha_{A}\right) \tau_{A}+d_{A} \alpha_{A} \tau_{A} .
$$


Each $\tau \in \Omega^{1}(M, a d P)$ defines the operator $B_{\tau} \phi:=[\tau, \phi]$ with $\phi \in \Omega^{q}(M, a d P)$. The curvature $\mathcal{F}$ of $\alpha$ reads

$$
\mathcal{F}_{A}\left(\tau_{1}, \tau_{2}\right)=\left(d_{A}^{*} d_{A}\right)^{-1}\left(B_{\tau_{1}^{h}}^{*} \tau_{2}^{h}-B_{\tau_{2}^{h}}^{*} \tau_{1}^{h}\right),
$$

where $B_{\tau}^{*}$ denotes the adjoint of $B_{\tau}$.

Atiyah and Singer introduced the universal bundle with a canonical connection in order to determine the characteristic classes of the index bundle of the family of Dirac operators coupled to gauge fields. The first Chern class turned out to represent an obstruction to the existence of a non vanishing gauge invariant determinant for fermions in the background of external Yang-Mills fields. A detailed review of the relation between index theory and non-abelian anomalies can be found in [Tr]. Now we shall briefly recall the construction of the universal bundle.

Consider the $G$-bundle $\mathcal{A} \times P \stackrel{\pi}{\rightarrow} \mathcal{A} \times M$, denoted by $\mathcal{Q}$, with principal action $R_{g}(A, p):=(A, p g)$. There exists a free right $\mathcal{G}$-action on $\mathcal{Q}$, given by $\mathcal{R}_{u}(A, p):=$ $\left(u^{*} A, u^{-1}(p)\right)$ so that the quotient $(\mathcal{A} \times P) / \mathcal{G}$ becomes a principal $G$ bundle $\hat{\mathcal{Q}}$ with base space $\mathcal{M} \times M$. The bundle $\hat{\mathcal{Q}}$ is called the universal bundle of Yang-Mills theory. We can summarize this construction by the commutative diagram

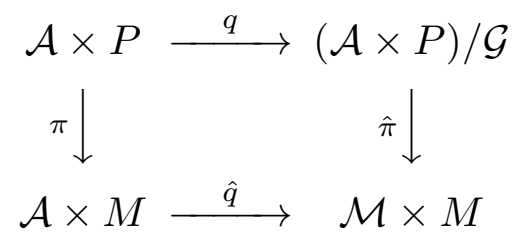

Obviously, one has $\hat{q}^{*} \hat{\mathcal{Q}} \cong \mathcal{Q}$. The algebra $\Omega(\mathcal{A} \times P)$ of differential forms on $\mathcal{A} \times P$ admits a bigrading $\Omega(\mathcal{A} \times P)=\oplus_{i, j \geq 0} \Omega^{(i, j)}(\mathcal{A} \times P)$. Thus a form $\phi \in \Omega^{(i, j)}(\mathcal{A} \times P)$ will be denoted by $\phi^{(i, j)}$.

There exists a canonical connection on $\mathcal{Q}$, defined by

$$
\gamma_{(A, p)}\left(\tau_{A}, X_{p}\right)=\gamma_{(A, p)}^{(0,1)}\left(X_{p}\right)+\gamma_{(A, p)}^{(1,0)}\left(\tau_{A}\right):=A_{p}\left(X_{p}\right)+\left(\alpha_{A}\left(\tau_{A}\right)\right)(p),
$$

where $X_{p} \in T_{p} P, \tau_{A} \in T_{A} \mathcal{A}$. It can be shown that $\gamma$ is $\mathcal{G}$-invariant. The vertical bundle $V^{q}(\mathcal{A} \times P)$ of the principal $\mathcal{G}$-bundle $\mathcal{A} \times P \stackrel{q}{\rightarrow}(\mathcal{A} \times P) / \mathcal{G}$ is given by

$$
V^{q}(\mathcal{A} \times P)=\bigcup_{(A, p) \in \mathcal{A} \times P}\left\{\left(d_{A} \xi,-Z_{\xi}(p)\right) \in T_{(A, p)}(\mathcal{A} \times P) \mid \xi \in \text { Lie } \mathcal{G}\right\},
$$

where $Z_{\xi}$ denotes the fundamental vector field on $P$ generated by $\xi$. Since $\left.\gamma\right|_{V^{q}(\mathcal{A} \times P)}=$ 0 , the connection $\gamma$ descends to a connection $\hat{\gamma}$ on the universal bundle, so that $\gamma=q^{*} \hat{\gamma}$. It has been shown in [AS] that this connection is universal for any family of connections on $P$ which is parametrized by any compact space.

The curvature $\Omega$ of $\gamma$ can be calculated by the formula $\Omega=d_{\mathcal{A} \times P} \gamma+\frac{1}{2}[\gamma, \gamma]$. Here, $d_{\mathcal{A} \times P}=d_{\mathcal{A}}+\hat{d}_{P}$, where $\hat{d}_{P}:=(-1)^{i} d_{P}: \Omega^{(i, j)}(\mathcal{A} \times P) \rightarrow \Omega^{(i, j+1)}(\mathcal{A} \times P)$. Being a two form on $\mathcal{A} \times P, \Omega$ decomposes into three components which are given by

$$
\begin{aligned}
\Omega_{(A, p)}^{(0,2)}\left(X_{p}^{1}, X_{p}^{2}\right) & =\left(F_{A}\right)_{p}\left(X_{p}^{1}, X_{p}^{2}\right) \\
\Omega_{(A, p)}^{(2,0)}\left(\tau_{A}^{1}, \tau_{A}^{2}\right) & =\left(d_{\mathcal{A}} \alpha+\frac{1}{2}[\alpha, \alpha]\right)_{(A, p)}\left(\tau_{A}^{1}, \tau_{A}^{2}\right)=\left(\mathcal{F}_{A}\left(\tau_{A}^{1}, \tau_{A}^{2}\right)\right)(p) \\
\Omega_{(A, p)}^{(1,1)}\left(\tau_{A}, X_{p}\right) & =\left(d_{\mathcal{A}} \gamma^{(0,1)}-d_{P} \gamma^{(1,0)}-\left[\gamma^{(0,1)}, \gamma^{(1,0)}\right]\right)_{(A, p)}\left(\tau_{A}, X_{p}\right) \\
& =\left(\tau_{A}\right)_{p}-\left(d_{A} \alpha_{A}\left(\tau_{A}\right)\right)_{p}\left(X_{p}\right)=\left(\tau_{A}^{h}\right)_{p}\left(X_{p}\right),
\end{aligned}
$$


where we used that

$$
\left(d_{\mathcal{A}} \gamma^{(0,1)}\right)_{(A, p)}\left(\tau_{A}, X_{p}\right)=\left.\frac{d}{d t}\right|_{t=0} \gamma_{\left(A+t \tau_{A}, p\right)}^{(0,1)}\left(X_{p}\right)=\left(\tau_{A}\right)_{p}\left(X_{p}\right)
$$

By construction, $\Omega$ descends to the curvature $\hat{\Omega}$ of $\hat{\gamma}$.

The BRS relations can now be obtained in the following way: Let

$$
\bar{i}_{A}: \mathcal{G} \times P \hookrightarrow \mathcal{A} \times P, \quad \bar{i}_{A}(u, p)=\left(i_{A} \times i d_{P}\right)(u, p)=\left(u^{*} A, p\right)
$$

be the embedding of a gauge orbit through $A \in \mathcal{A}$. We denote the Maurer Cartan form on $\mathcal{G}$ by $\Theta \in \Omega^{1}(\mathcal{G}$, Lie $\mathcal{G})$. Any tangent vector $\mathcal{Y}_{u} \in T_{u} \mathcal{G}$ can be written in the form $\mathcal{Y}_{u}=\mathcal{Y}_{\Theta_{u}\left(\mathcal{Y}_{u}\right)}^{\text {left }}(u)$, where $\mathcal{Y}_{\Theta_{u}\left(\mathcal{Y}_{u}\right)}^{\text {left }}$ denotes the left invariant vector field on $\mathcal{G}$ induced by $\Theta_{u}\left(\mathcal{Y}_{u}\right) \in$ Lie $\mathcal{G}$. Then the restriction of $\gamma$ to the gauge orbit through $A \in \mathcal{A}$ reads

$\left(\bar{i}_{A}^{*} \gamma\right)_{(u, p)}\left(\mathcal{Y}_{u}, X_{p}\right)=\left(\bar{i}_{A}^{*} \gamma\right)_{(u, p)}^{(0,1)}\left(X_{p}\right)+\left(\bar{i}_{A}^{*} \gamma\right)_{(u, p)}^{(1,0)}\left(\mathcal{Y}_{u}\right)=\left(u^{*} A\right)_{p}\left(X_{p}\right)+\left(\Theta_{u}\left(\mathcal{Y}_{u}\right)\right)(p)$

Furthermore the restriction of the curvature gives the result

$$
\left(\bar{i}_{A}^{*} \Omega\right)_{(u, p)}\left(\left(\mathcal{Y}_{u}^{1}, X_{p}^{1}\right),\left(\mathcal{Y}_{u}^{2}, X_{p}^{2}\right)\right)=\left(u^{*} F_{A}\right)_{p}\left(X_{p}^{1}, X_{p}^{2}\right),
$$

which is the so-called "Russian formula" [S]. There is a one to one correspondence between this formula and the BRS relations. In fact, setting $\bar{A}:=\left(\bar{i}_{A}^{*} \gamma\right)^{(0,1)}$ and $\bar{F}_{A}=\bar{i}_{A}^{*} \Omega$ we obtain from (2.9) and (2.13)

$$
\begin{aligned}
\bar{F}_{A} & =F_{\bar{A}}=d_{P} \bar{A}+\frac{1}{2}[\bar{A}, \bar{A}] \\
0 & =d_{\mathcal{G}} \Theta+\frac{1}{2}[\Theta, \Theta] \\
0 & =d_{\mathcal{G}} \bar{A}-d_{\bar{A}} \Theta .
\end{aligned}
$$

On the other hand, the Bianchi identity for $\bar{i}_{A}^{*} \Omega$ yields

$$
d_{\mathcal{G}}\left(\bar{F}_{A}\right)+\left[\Theta, \bar{F}_{A}\right]=0 \text {. }
$$

These are the BRS relations $[\mathrm{S}]$, where $d_{\mathcal{G}}$ and $\Theta$ are identified with the BRS operator and the ghost respectively. (The minus in the last equation of (2.14) occurs because in our convention $d_{\mathcal{G}}$ and $d_{P}$ commute with each other.) Thus we have explicitly verified that the bundle $\mathcal{Q}$ provides an appropriate geometrical framework to realize the BRS multiplet.

Let $I^{m}(G)$ denote the space of ad-invariant, symmetric multilinear real valued functions on $\mathfrak{g}$ of degree $m$. For a given $Q \in I^{m}(G)$ with $m>\frac{n}{2}$, the consistent descent equations can be derived from the transgression formula $[\mathrm{C}]$

$$
Q(\Omega)=d_{\mathcal{A} \times P} T Q(\gamma)
$$

where $T Q(\gamma)=m \int_{0}^{1} d t Q\left(\gamma, \Omega_{t}, \ldots, \Omega_{t}\right)$ with $\Omega_{t}=t \Omega+\frac{t^{2}-t}{2}[\gamma, \gamma]$. Explicitly, the components of $\Omega_{t}$ are given by

$$
\begin{aligned}
& \Omega_{t(A, p)}^{(0,2)}\left(X_{p}^{1}, X_{p}^{2}\right)=\left(\left(F_{A}\right)_{t}\right)_{p}\left(X_{p}^{1}, X_{p}^{2}\right)=\left(t F_{A}+\frac{t^{2}-t}{2}[A, A]\right)_{p}\left(X_{p}^{1}, X_{p}^{2}\right) \\
& \Omega_{t(A, p)}^{(1,1)}\left(\tau_{A}, X_{p}\right)=\left(t \Omega_{(A, p)}^{(1,1)}+\left(t^{2}-t\right)\left[\alpha_{A}, A\right]\right)\left(\tau_{A}, X_{p}\right) \\
& \Omega_{t} \underset{(A, p)}{(2,0)}\left(\tau_{A}^{1}, \tau_{A}^{2}\right)=\left(\mathcal{F}_{t}\right)_{A}\left(\tau_{A}^{1}, \tau_{A}^{2}\right)(p)=\left(t \mathcal{F}+\frac{t^{2}-t}{2}[\alpha, \alpha]\right)_{A}\left(\tau_{A}^{1}, \tau_{A}^{2}\right)(p)
\end{aligned}
$$


With respect to the product structure of $\Omega(\mathcal{A} \times P),(2.16)$ gives

$$
Q(\Omega)^{(k, 2 m-k)}=d_{\mathcal{A}} T Q(\gamma)^{(k-1,2 m-k)}+\hat{d}_{P} T Q(\gamma)^{(k, 2 m-k-1)} \quad 0 \leqq k \leqq 2 m
$$

The restriction of (2.18) to a gauge orbit through $A \in \mathcal{A}$ finally leads to the well known consistent descent equations $[\mathrm{S}, \mathrm{Zu}]$

$$
\begin{aligned}
Q\left(u^{*} F_{A}\right) & =d_{P} T Q\left(u^{*} A\right)^{(0,2 m-1)}=d_{P} T Q\left(\bar{i}_{A}^{*} \gamma\right)^{(0,2 m-1)} \\
0 & =d_{\mathcal{G}} T Q\left(\bar{i}_{A}^{*} \gamma\right)^{(k-1,2 m-k)}+\hat{d}_{P} T Q\left(\bar{i}_{A}^{*} \gamma\right)^{(k, 2 m-k-1)} \quad 1 \leqq k \leqq 2 m
\end{aligned}
$$

The relation for $k=2$ is the Wess-Zumino consistency condition [WZ]. Explicitly,

$$
\begin{aligned}
&\left.T Q\left(\bar{i}_{A}^{*} \gamma\right)^{(1,2 m-2)}\right|_{u=i d_{\mathcal{G}}}(\xi) \\
&= m \int_{0}^{1} d t\left\{Q\left(\xi,\left(F_{A}\right)_{t}, \ldots,\left(F_{A}\right)_{t}\right)+(m-1) \frac{t^{2}-t}{2} Q\left(A,[A, \xi],\left(F_{A}\right)_{t}, \ldots,\left(F_{A}\right)_{t}\right)\right\} \\
&(2.20)
\end{aligned}
$$

gives the non-integrated consistent current anomaly [ZWZ] and $\left.T Q\left(\bar{i}_{A}^{*} \gamma\right)^{(2,2 m-3)}\right|_{u=i d_{\mathcal{G}}}$ may be identified with the non-integrated consistent Schwinger term $[\mathrm{F}, \mathrm{M}]$.

For $n=2 m-2$, one finds that $Q(\Omega)^{(0, n+2)}=0$ for dimensional reasons and therefore $T Q\left(\bar{i}_{A}^{*} \gamma\right)^{(0, n+1)}$ is $d_{P}$ closed on $\mathcal{G} \times P$, inducing the de Rham cohomology class

$$
\left[T Q\left(\bar{i}_{A}^{*} \gamma\right)^{(0, n+1)}\right] \in H_{d_{P}}^{(0, n+1)}(\mathcal{G} \times P, \mathbb{R}) .
$$

This result has been firstly obtained in [BC1].

So far we have expressed the consistent anomalous terms by differential forms on the space $\mathcal{G} \times P$. It is often convenient to express them in terms of forms on the base $\mathcal{G} \times M$. So we choose a fixed connection $a \in \mathcal{A}$ and extend it to a connection

$$
\gamma_{(A, p)}^{a}\left(\tau_{A}, X_{p}\right):=a_{p}\left(X_{p}\right)
$$

in the $G$-bundle $\mathcal{A} \times P \rightarrow \mathcal{A} \times M$. Its curvature $\Omega^{a}$ is given by $\Omega^{a}=F_{a}$ and therefore $Q\left(\Omega^{a}\right)=Q\left(F_{a}\right)=0$ because of dimensional reasons. Following [MSZ], we replace $(2.16)$ by

$$
Q(\Omega)=Q(\Omega)-Q\left(\Omega^{a}\right)=d_{\mathcal{A} \times P} T Q\left(\gamma, \gamma^{a}\right)
$$

with $T Q\left(\gamma, \gamma^{a}\right)=m \int_{0}^{1} d t Q\left(\gamma-\gamma^{a}, \Omega_{t}^{\prime}, \ldots, \Omega_{t}^{\prime}\right)$ where $\Omega_{t}^{\prime}$ is the curvature of the connection $\gamma_{t}=t \gamma+(1-t) \gamma^{a}$. The transgression form $T Q\left(\gamma, \gamma^{a}\right)$ is basic and thus projects to a $(2 m-1)$-form $\overline{T Q\left(\gamma, \gamma^{a}\right)}$ on $\mathcal{A} \times M$.

Applying the pullback $\bar{i}_{A}^{*}$ to $(2.23)$, one is left with

$$
\begin{aligned}
Q\left(u^{*} F_{A}\right) & =d_{P} T Q\left(\bar{i}_{A}^{*} \gamma, \bar{i}_{A}^{*} \gamma^{a}\right)^{(0,2 m-1)} \\
0 & =d_{\mathcal{G}} T Q\left(\bar{i}_{A}^{*} \gamma, \bar{i}_{A}^{*} \gamma^{a}\right)^{(k-1,2 m-k)}+\hat{d}_{P} T Q\left(\bar{i}_{A}^{*} \gamma, \bar{i}_{A}^{*} \gamma^{a}\right)^{(k, 2 m-k-1)} \quad 1 \leq k \leq 2 m
\end{aligned}
$$

Since the following identity holds

$$
T Q\left(\gamma, \gamma^{a}\right)=T Q(\gamma)-T Q\left(\gamma^{a}\right)+d_{\mathcal{A} \times P} S_{Q}\left(\gamma, \gamma^{a}\right)
$$


where

$$
S_{Q}\left(\gamma, \gamma^{a}\right)=m(m-1) \int_{0}^{1} d t_{1} \int_{0}^{1} d t_{2} t_{2} Q\left(\gamma-\gamma^{a}, \gamma_{t_{1}},(\Omega)_{t_{2}}^{\left(t_{1}\right)}, \ldots,(\Omega)_{t_{2}}^{\left(t_{1}\right)}\right)
$$

with $\gamma_{t_{1}}=t_{1} \gamma+\left(1-t_{1}\right) \gamma^{a}$ and $(\Omega)_{t_{2}}^{\left(t_{1}\right)}=t_{2} d_{\mathcal{A} \times P} \gamma_{t_{1}}+\frac{t_{2}^{2}}{2}\left[\gamma_{t_{1}}, \gamma_{t_{1}}\right]$, we can explicitly verify that $T Q\left(\bar{i}_{A}^{*} \gamma, \bar{i}_{A}^{*} \gamma^{a}\right)^{(k, 2 m-k-1)}$ and $T Q\left(\bar{i}_{A}^{*} \gamma\right)^{(k, 2 m-k-1)}$ represent the same cohomology class with respect to the cohomology $d_{\mathcal{G}}$ modulo $d_{P}$, i.e. they are cohomologous with respect to the BRS-cohomology.

Let $N$ be a $(2 m-k-1)$-dimensional, submanifold of $M$ without boundary, then the integrated consistent anomaly $\mathfrak{A}^{(k)}$ of ghost degree $k$ is identified with

$$
\begin{aligned}
\mathfrak{A}^{(k)}\left(A, \xi_{1}, \ldots, \xi_{k}\right) & :=\left.\int_{N} \overline{T Q\left(\bar{i}_{A}^{*} \gamma, \bar{i}_{A}^{*} \gamma^{a}\right)}{ }^{(k, 2 m-k-1)}\right|_{u=i d_{\mathcal{G}}}\left(\xi_{1}, \ldots, \xi_{k}\right) \\
& =\left(i_{Z_{\xi_{1}}^{\mathcal{A}}} \cdots i_{Z_{\xi_{k}}^{\mathcal{A}}} \int_{N}{\overline{T Q\left(\gamma, \gamma^{a}\right)}}^{(k, 2 m-k-1)}\right)(A) .
\end{aligned}
$$

It defines, for variable $A \in \mathcal{A}$, a class of degree $k$ in the Lie algebra cohomology of Lie $\mathcal{G}$ with values in $C^{\infty}(\mathcal{A})[\mathrm{BC} 2]$.

\section{The COVARIANT DESCEnT EQUATIONS}

In this section we want to show that the bundle $\mathcal{Q}$ also provides an appropriate framework to formulate descent equations for covariant anomalies.

We define a connection on $\mathcal{Q}$ by

$$
\left(\gamma_{0}\right)_{(A, p)}\left(\tau_{A}, X_{p}\right):=A_{p}\left(X_{p}\right)
$$

It is easily shown that this connection is $\mathcal{G}$-invariant. However, it does not induce

a connection on $\hat{\mathcal{Q}}$. The curvature $\Omega_{0}$ of $\gamma_{0}$ decomposes into the three components

$$
\begin{aligned}
& \Omega_{0} \stackrel{(2,0)}{(2)}=0 \\
& \Omega_{0} \stackrel{(1,1)}{(A, p)}\left(\tau_{A}, X_{p}\right)=\left(d_{\mathcal{A}} A\right)_{p}\left(\tau_{A}, X_{p}\right)=\left(\tau_{A}\right)_{p}\left(X_{p}\right) \\
& \Omega_{0} \stackrel{(0,2)}{(A, p)}\left(X_{p}^{1}, X_{p}^{2}\right)=\left(F_{A}\right)_{p}\left(X_{p}^{1}, X_{p}^{2}\right) .
\end{aligned}
$$

Now we want to derive descent equations for the connection $\gamma_{0}$. Let $Q \in I^{m}(G)$, then the equations $d_{\mathcal{A} \times P} Q\left(\Omega_{0}\right)=0$ and $Q\left(\Omega_{0}\right)=d_{\mathcal{A} \times P} T Q\left(\gamma_{0}\right)$ imply

$$
\begin{aligned}
0 & =d_{\mathcal{A}} Q\left(\Omega_{0}\right)^{(k-1,2 m-k+1)}+\hat{d}_{P} Q\left(\Omega_{0}\right)^{(k, 2 m-k)} & & 0 \leqq k \leqq m \\
Q\left(\Omega_{0}\right)^{(k, 2 m-k)} & =d_{\mathcal{A}} T Q\left(\gamma_{0}\right)^{(k-1,2 m-k)}+\hat{d}_{P} T Q\left(\gamma_{0}\right)^{(k, 2 m-k-1)} & & 0 \leqq k \leqq m
\end{aligned}
$$

The components of $\left(\Omega_{0}\right)_{t}:=t \Omega_{0}+\frac{t^{2}-t}{2}\left[\gamma_{0}, \gamma_{0}\right]$ are

$$
\begin{aligned}
& \left(\Omega_{0}\right)_{t} \stackrel{(2,0)}{ }=0 \\
& \left(\Omega_{0}\right)_{t} \stackrel{(1,1)}{(A, p)}\left(\tau_{A}, X_{p}\right)=t d_{\mathcal{A}} A\left(\tau_{A}, X_{p}\right)=t\left(\tau_{A}\right)_{p}\left(X_{p}\right) \\
& \left(\Omega_{0}\right)_{t} \stackrel{(0,2)}{(A, p)}\left(X_{p}^{1}, X_{p}^{2}\right)=\left(\left(F_{A}\right)_{t}\right)_{p}\left(X_{p}^{1}, X_{p}^{2}\right)
\end{aligned}
$$


Thus it is evident from (3.2) and (3.4) that $Q\left(\Omega_{0}\right)^{(k, 2 m-k)}=0$ for $k \geq m+1$ and $T Q\left(\gamma_{0}\right)^{(k, 2 m-k-1)}=0$ for $k \geq m$. However, we should mention that there is no analog of the Russian formula for $\Omega_{0}$. In fact, the restriction of $\Omega_{0}$ to a gauge orbit yields

$$
\begin{aligned}
& \left(\bar{i}_{A}^{*} \Omega_{0}^{(2,0)}\right)=0 \\
& \left(\bar{i}_{A}^{*} \Omega_{0}^{(1,1)}\right)_{(u, p)}\left(\mathcal{Y}_{u}, X_{p}\right)=\left(d_{u^{*} A} \Theta_{u}\left(\mathcal{Y}_{u}\right)\right)_{p}\left(X_{p}\right) \\
& \left(\bar{i}_{A}^{*} \Omega_{0}^{(0,2)}\right)_{(u, p)}\left(X_{p}^{1}, X_{p}^{2}\right)=\left(u^{*} F_{A}\right)_{p}\left(X_{p}^{1}, X_{p}^{2}\right),
\end{aligned}
$$

where the BRS relations (2.14) have been used.

If we define the secondary characteristic form $T Q\left(\gamma, \gamma_{0}\right) \in \Omega^{2 m-1}(\mathcal{A} \times P)$ by

$$
Q(\Omega)-Q\left(\Omega_{0}\right)=d_{\mathcal{A} \times P} T Q\left(\gamma, \gamma_{0}\right)
$$

the corresponding set of descent equations reads

$$
Q(\Omega)^{(k, 2 m-k)}-Q\left(\Omega_{0}\right)^{(k, 2 m-k)}=d_{\mathcal{A}} T Q\left(\gamma, \gamma_{0}\right)^{(k-1,2 m-k)}+\hat{d}_{P} T Q\left(\gamma, \gamma_{0}\right)^{(k, 2 m-k-1)} .
$$

The curvature $\Omega_{t}^{\prime}$ of the interpolating connection $t \gamma+(1-t) \gamma_{0}$ has the components

$$
\begin{aligned}
& \Omega_{t}^{\prime(A, p)}\left(\tau_{A}^{1}, \tau_{A}^{2}\right)=\left(\mathcal{F}_{t}\right)_{A}\left(\tau_{A}^{1}, \tau_{A}^{2}\right)(p) \\
& \Omega_{t(A, p)}^{\prime(1,1)}\left(\tau_{A}, X_{p}\right)=\left(d_{\mathcal{A}} A-t d_{A} \alpha_{A}\right)\left(\tau_{A}, X_{p}\right)=\left(\tau_{A}-t d_{A} \alpha_{A}\left(\tau_{A}\right)\right)_{p}\left(X_{p}\right) \\
& \Omega_{t}^{\prime(A, p)}\left(X_{p}^{1}, X_{p}^{2}\right)=\left(F_{A}\right)_{p}\left(X_{p}^{1}, X_{p}^{2}\right) \text {. }
\end{aligned}
$$

Finally, the restriction to a gauge orbit in $\mathcal{A}$ leads to the generalized descent equations

$$
-Q\left(\bar{i}_{A}^{*} \Omega_{0}\right)^{(k, 2 m-k)}=d_{\mathcal{G}} T Q\left(\bar{i}_{A}^{*} \gamma, \bar{i}_{A}^{*} \gamma_{0}\right)^{(k-1,2 m-k)}+\hat{d}_{P} T Q\left(\bar{i}_{A}^{*} \gamma, \bar{i}_{A}^{*} \gamma_{0}\right)^{(k, 2 m-k-1)},
$$

where $1 \leq k \leq 2 m$. In order to prove the relevance of our construction for covariant anomalies we calculate, using (3.8)

$$
\left.T Q\left(\bar{i}_{A}^{*} \gamma, \bar{i}_{A}^{*} \gamma_{0}\right)^{(1,2 m-2)}\right|_{u=i d_{\mathcal{G}}}=m Q\left(\Theta, F_{A}, \ldots, F_{A}\right)
$$

which is the non-integrated covariant anomaly [BZ]. For $k=2$, we obtain

$$
\left.T Q\left(\bar{i}_{A}^{*} \gamma, \bar{i}_{A}^{*} \gamma_{0}\right)^{(2,2 m-3)}\right|_{u=i d_{\mathcal{G}}}=\frac{m(m-1)}{2} Q\left(\Theta, d_{A} \Theta, F_{A}, \ldots, F_{A}\right)
$$

This result agrees with previous calculations of the non-integrated covariant Schwinger term [Ts, AAG]. Thus we have derived a new set of descent equations which contains the covariant current anomaly and the covariant Schwinger term. The differential forms

$$
Q\left(\Omega_{0}\right)_{(A, p)}^{(k, 2 m-k)}=\left(\begin{array}{c}
m \\
k
\end{array}\right) Q(\underbrace{d_{\mathcal{A}} A, \ldots, d_{\mathcal{A}} A}_{k \text { times }}, \underbrace{F_{A}, \ldots, F_{A}}_{m-k \text { times }})(p)
$$

vanish for $k \leq m-1$ if and only if $A$ is a flat connection. In general, the forms $T Q\left(\bar{i}_{A}^{*} \gamma, \bar{i}_{A}^{*} \gamma_{0}\right)^{(k-1,2 m-k)}$ do not define cohomology classes $d_{\mathcal{G}}$ modulo $d_{P}$. Although 
this fact is well known for the cases $k=1,2$, we have explicitly identified its geometrical origin.

Now we want to study the properties of $T Q\left(\gamma, \gamma_{0}\right)$ under gauge transformations. Therefore let $\overline{T Q\left(\gamma, \gamma_{0}\right)}$ denote the projection of the transgression form $T Q\left(\gamma, \gamma_{0}\right)$ onto $\mathcal{A} \times M$. Since $T Q\left(\gamma, \gamma_{0}\right)$ is $\mathcal{G}$ invariant with respect to $\mathcal{R}, \pi \circ \mathcal{R}_{u}=\left(\mathcal{R}_{u}^{\mathcal{A}} \times\right.$ $\left.i d_{M}\right) \circ \pi$ implies

$$
\left(\mathcal{R}_{u}^{\mathcal{A}} \times i d_{M}\right)^{*} \overline{T Q\left(\gamma, \gamma_{0}\right)}=\overline{T Q\left(\gamma, \gamma_{0}\right)} .
$$

Since $\pi \circ\left(\mathcal{R}_{u}^{\mathcal{A}} \times i d_{P}\right)=\left(\mathcal{R}_{u}^{\mathcal{A}} \times i d_{M}\right) \circ \pi$, one finally obtains

$$
\left(\mathcal{R}_{u}^{\mathcal{A}} \times i d_{P}\right)^{*} T Q\left(\gamma, \gamma_{0}\right)=T Q\left(\gamma, \gamma_{0}\right)
$$

Let $\wedge L i e \mathcal{G}^{*}$ denote the exterior algebra of the dual of the gauge algebra and let $a d^{*}$ denote the coadjoint action of $\mathcal{G}$ in $L_{i e} \mathcal{G}^{*}$. We define the map

$$
\begin{aligned}
& h^{(k)}: \Omega^{(k, 2 m-k-1)}(\mathcal{A} \times P) \rightarrow \Omega^{(0,2 m-k-1)}\left(\mathcal{A} \times P, \wedge^{k} L_{i e} \mathcal{G}^{*}\right) \\
& \left(h^{(k)} \phi^{(k, 2 m-k-1)}\right)_{(A, p)}\left(X_{1}, \ldots, X_{2 m-k-1}\right)\left(\xi_{1}, \ldots, \xi_{k}\right):= \\
& \phi_{(A, p)}^{(k, 2 m-k-1)}\left(Z_{\xi_{1}}^{\mathcal{A}}(A), \ldots, Z_{\xi_{k}}^{\mathcal{A}}(A), X_{1}, \ldots, X_{2 m-k-1}\right),
\end{aligned}
$$

where $X_{i} \in T_{p} P$ and $\xi_{i} \in L i e \mathcal{G}$. The form $\chi^{(k, 2 m-k-1)}:=h^{(k)} T Q\left(\gamma, \gamma_{0}\right)^{(k, 2 m-k-1)}$ is basic with projection $\bar{\chi}^{(k, 2 m-k-1)}$. We have now the following

3.16 Proposition. $\left(\mathcal{R}_{u}^{\mathcal{A}} \times i d_{P}\right)^{*} \chi^{(k, 2 m-k-1)}=a d^{*}\left(\hat{u}^{-1}\right) \chi^{(k, 2 m-k-1)}$.

Proof. Using (3.14), one finds

$$
\begin{aligned}
& \left(\left(\mathcal{R}_{u}^{\mathcal{A}} \times i d_{P}\right)^{*} \chi^{(k, 2 m-k-1)}\right)_{(A, p)}\left(X_{1}, \ldots, X_{2 m-k-1}\right)\left(\xi_{1}, \ldots, \xi_{k}\right) \\
& \quad=\left(T Q\left(\gamma, \gamma_{0}\right)^{(k, 2 m-k-1)}\right)_{\left(u^{*} A, p\right)}\left(T_{A} \mathcal{R}_{u}^{\mathcal{A}} Z_{a d(u) \xi_{1}}^{\mathcal{A}}, \ldots, T_{A} \mathcal{R}_{u}^{\mathcal{A}} Z_{a d(u) \xi_{k}}^{\mathcal{A}}, X_{1}, \ldots, X_{2 m-k-1}\right) \\
& \quad=\left(\chi^{(k, 2 m-k-1)}\right)_{\left(u^{*} A, p\right)}\left(X_{1}, \ldots, X_{2 m-k-1}\right)\left(a d(u) \xi_{1}, \ldots, a d(u) \xi_{k}\right) .
\end{aligned}
$$

Let $N$ be a $(2 m-k-1)$-dimensional, submanifold of $M$ without boundary then we define the covariant anomaly $\tilde{\mathfrak{A}}^{(k)}$ of ghost degree $k$ by

$$
\begin{aligned}
\tilde{\mathfrak{A}}^{(k)}\left(A, \xi_{1}, \ldots, \xi_{k}\right) & :=\left.\int_{N} \overline{T Q\left(\bar{i}_{A}^{*} \gamma, \bar{i}_{A}^{*} \gamma_{0}\right)}{ }^{(k, 2 m-k-1)}\right|_{u=i d_{\mathcal{G}}}\left(\xi_{1}, \ldots, \xi_{k}\right) \\
& =\left(i_{Z_{\xi_{1}}^{\mathcal{A}}} \cdots i_{Z_{\xi_{k}}^{\mathcal{A}}} \int_{N} \overline{T Q\left(\gamma, \gamma_{0}\right)}{ }^{(k, 2 m-k-1)}\right)(A) .
\end{aligned}
$$

Regarding $\tilde{\mathfrak{A}}^{(k)}$ as a map $\mathcal{A} \rightarrow \wedge^{k} L i e \mathcal{G}^{*}$, one has $\tilde{\mathfrak{A}}^{(k)}=\int_{N} \bar{\chi}^{(k, 2 m-k-1)}$. Let $\wedge^{k} a d^{*} \mathcal{A}=\mathcal{A} \times{ }_{\mathcal{G}} \wedge^{k}{ }^{2 i e} \mathcal{G}^{*}$ denote the bundle over $\mathcal{M}$ associated to $\mathcal{A} \rightarrow \mathcal{M}$ with respect to the coadjoint action then the above proposition implies that $\tilde{\mathfrak{A}}^{(k)}$ induces a section of $\wedge^{k} a d^{*} \mathcal{A}$. This result has been obtained in a different way in [K1]. Since the Lie derivative of $\tilde{\mathfrak{A}}^{(k)}$ yields

$$
\left(L_{Z_{\xi}^{\mathcal{A}}} \tilde{\mathfrak{A}}^{(k)}\right)(A)\left(\xi_{1}, \ldots, \xi_{k}\right)=\sum_{i=1}^{k} \tilde{\mathfrak{A}}^{(k)}\left(A, \xi_{1}, \ldots,\left[\xi, \xi_{i}\right], \ldots, \xi_{k}\right),
$$


one finally obtains, using (3.7)

$$
\begin{aligned}
i_{Z_{\xi_{1}}^{\mathcal{A}}} \cdots i_{Z_{\xi_{k}}^{\mathcal{A}}} \int_{N} & \overline{Q\left(\Omega_{0}\right)}(k, 2 m-k) \\
& =-\sum_{1 \leq i<j \leq k}(-1)^{(i+j)} \tilde{\mathfrak{A}}^{(k)}\left(A,\left[\xi_{i}, \xi_{j}\right], \xi_{1} \ldots, \hat{\xi}_{i}, \ldots, \hat{\xi}_{j}, \ldots, \xi_{k}\right),
\end{aligned}
$$

where the caret denotes omission of the corresponding element.

In the remainder of this section we want to investigate the relation between consistent and covariant terms in more detail. In Sec. 2 the consistent anomalies have been identified with the family of forms $T Q\left(\bar{i}_{A}^{*} \gamma\right)^{(k, 2 m-k-1)}$. Because of the identity

$$
T Q\left(\gamma, \gamma_{0}\right)=T Q(\gamma)-T Q\left(\gamma_{0}\right)+d_{\mathcal{A} \times P} S_{Q}\left(\gamma, \gamma_{0}\right)
$$

the general counterterm relating the non-integrated consistent and covariant anomalies is given by

$$
\begin{aligned}
\lambda\left(\gamma, \gamma_{0}\right)^{(k, 2 m-k-1)}:= & T Q\left(\gamma_{0}\right)^{(k, 2 m-k-1)}-d_{\mathcal{A}} S_{Q}\left(\gamma, \gamma_{0}\right)^{(k-1,2 m-k-1)} \\
& +(-1)^{k+1} d_{P} S_{Q}\left(\gamma, \gamma_{0}\right)^{(k, 2 m-k-2)}
\end{aligned}
$$

Up to a $d_{P}$ exact form, the counterterm for $k=1$ is given by

$$
T Q\left(\gamma_{0}\right)^{(1,2 m-2)}=m(m-1) \int_{0}^{1} d t t Q\left(A, d_{\mathcal{A}} A,\left(F_{A}\right)_{t}, \ldots,\left(F_{A}\right)_{t}\right),
$$

where (3.4) has been used. This is the expression for the non-integrated BardeenZumino functional [BZ] in dimension $2 m-2$. In order to find the corresponding expressions for the integrated anomalies we shall use the background connection $\gamma^{a}$ of (2.22). The secondary characteristic forms corresponding to the connections $\gamma$, $\gamma_{0}$ and $\gamma_{a}$ fulfill the following general identity ("triangle formula") [MSZ]

$$
T Q\left(\gamma, \gamma_{a}\right)+T Q\left(\gamma_{a}, \gamma_{0}\right)+T Q\left(\gamma_{0}, \gamma\right)=d_{\mathcal{A} \times P} S Q\left(\gamma^{a}, \gamma, \gamma_{0}\right)
$$

with

$$
S Q\left(\gamma^{a}, \gamma, \gamma_{0}\right)=m(m-1) \int_{t_{1}+t_{2} \leqq 1} d t_{1} d t_{2} Q\left(\gamma-\gamma_{0}, \gamma^{a}-\gamma_{0}, \Omega_{t_{1} t_{2}}, \ldots, \Omega_{t_{1} t_{2}}\right)
$$

where $\Omega_{t_{1} t_{2}}$ is the curvature of $\gamma_{0}+t_{1}\left(\gamma-\gamma_{0}\right)+t_{2}\left(\gamma^{a}-\gamma_{0}\right) . S Q\left(\gamma^{a}, \gamma, \gamma_{0}\right)$ is a basic $(2 m-2)$ form on $\mathcal{A} \times P$. Then the counterterm with background connection becomes

$$
\begin{aligned}
\lambda\left(\gamma, \gamma_{0}, \gamma_{a}\right)^{(k, 2 m-k-1)}:= & T Q\left(\gamma_{0}, \gamma_{a}\right)^{(k, 2 m-k-1)}-d_{\mathcal{A}} S Q\left(\gamma^{a}, \gamma, \gamma_{0}\right)^{(k-1,2 m-k-1)} \\
& +(-1)^{k+1} d_{P} S Q\left(\gamma^{a}, \gamma, \gamma_{0}\right)^{(k, 2 m-k-2)}
\end{aligned}
$$

which is a basic form with projection $\overline{\lambda\left(\gamma, \gamma_{0}, \gamma_{a}\right)}(k, 2 m-k-1) \in \Omega^{(k, 2 m-k-1)}(\mathcal{A} \times M)$.

Let $N$ be a $(2 m-k-1)$-dimensional submanifold of $M$ without boundary then the integrated consistent and covariant anomaly of ghost degree $k$ are related by a generalized Bardeen-Zumino relation

$$
\tilde{\mathfrak{A}}^{(k)}\left(\xi_{1}, \ldots, \xi_{k}\right)=\mathfrak{A}^{(k)}\left(\xi_{1}, \ldots, \xi_{k}\right)-i_{Z_{\xi_{1}}^{\mathcal{A}}} \cdots i_{Z_{\xi_{k}}^{\mathcal{A}}} \int_{N}{\overline{\lambda\left(\gamma, \gamma_{0}, \gamma_{a}\right)}}^{(k, 2 m-k-1)} .
$$


Since $Q\left(\Omega_{0}\right)=Q\left(\Omega_{0}\right)-Q\left(\Omega^{a}\right)=d_{\mathcal{A} \times P} T Q\left(\gamma_{0}, \gamma^{a}\right)$, one obtains using (3.25)

$$
\int_{N}{\overline{Q\left(\Omega_{0}\right)}}^{(k+1,2 m-k-1)}=d_{\mathcal{A}} \int_{N}{\overline{\lambda\left(\gamma, \gamma_{0}, \gamma^{a}\right)}}^{(k, 2 m-k-1)} .
$$

In order to make contact with the result of [BC1] we shall choose $n=\operatorname{dim} M=$ $2 m-2$. This implies

$$
0=Q\left(\bar{i}_{A}^{*} \Omega_{0}\right)^{(1, n+1)}=d_{P} T Q\left(\bar{i}_{A}^{*} \gamma, \bar{i}_{A}^{*} \gamma_{0}\right)^{(1, n)}=d_{P}\left(T Q\left(\bar{i}_{A}^{*} \gamma\right)^{(1, n)}-T Q\left(\bar{i}_{A}^{*} \gamma_{0}\right)^{(1, n)}\right)
$$

and by (3.21) we obtain

$$
\left[T Q\left(\bar{i}_{A}^{*} \gamma, \bar{i}_{A}^{*} \gamma_{0}\right)^{(1, n)}\right]=\left[T Q\left(\bar{i}_{A}^{*} \gamma\right)^{(1, n)}-T Q\left(\bar{i}_{A}^{*} \gamma_{0}\right)^{(1, n)}\right] \in H_{d_{P}}^{(1, n)}(\mathcal{G} \times P, \mathbb{R})
$$

Actually, $\left[\overline{T Q\left(\bar{i}_{A}^{*} \gamma, \bar{i}_{A}^{*} \gamma_{0}\right)}{ }^{(1, n)}\right] \in H_{d_{M}}^{(1, n)}(\mathcal{G} \times M, \mathbb{R})$ because the transgression form is basic.

Since the form $T Q\left(\bar{i}_{A}^{*} \gamma, \bar{i}_{A}^{*} \gamma_{0}\right)^{(1, n)}$ is local in the sense of [BC1], i.e. polynomial in the fields $A$ and $F_{A}$ and linear in the ghost, it finally gives a certain class in the local de-Rham cohomology $H_{d_{P}}^{(1, n)}(\mathcal{G} \times P, \mathbb{R})_{\text {loc }}$. So we have recovered the result of [BC1] as a direct consequence of the generalized descent equations.

\section{BRS, ANTI-BRS TRANSFORMATIONS AND THE COVARIANCE CONDITION}

In [Ts] and [Z] a covariance condition for covariant anomalies has been formulated in terms of the BRS/anti-BRS algebra. The aim of this section is to analyze the geometrical meaning of this condition. The first step will be the construction of a geometrical realization of the BRS/anti-BRS multiplet.

Let us consider the principal $G$-bundle $\mathcal{A} \times \mathcal{G} \times P \stackrel{\tilde{\pi}}{\rightarrow} \mathcal{A} \times \mathcal{G} \times M$, denoted by $\mathcal{P}$, with principal action $\tilde{R}_{g}(A, u, p)=(A, u, p g)$ and projection $\tilde{\pi}$. The map $\mathcal{R}^{\mathcal{A}} \times i d_{P}$ is a $G$ bundle homomorphism which makes the following diagram commutative

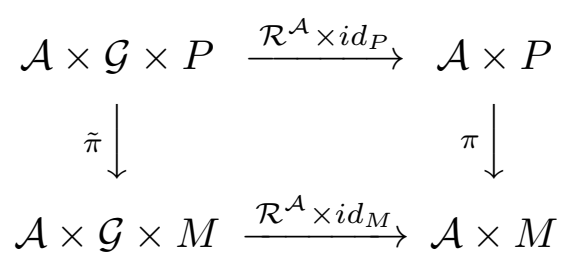

In fact, $\left(\mathcal{R}^{\mathcal{A}} \times i d_{M}\right)^{*} \mathcal{Q} \cong \mathcal{P}$. Here $\Omega(\mathcal{A} \times \mathcal{G} \times P)=\oplus_{i, j, k \geq 0} \Omega^{(i, j, k)}(\mathcal{A} \times \mathcal{G} \times P)$ admits a tripel grading. Hence we write $d_{\mathcal{A} \times \mathcal{G} \times P}=d_{\mathcal{A}}+\hat{d}_{\mathcal{G}}+\hat{d}_{P}$, where

$$
\begin{aligned}
& \hat{d}_{\mathcal{G}}:=(-1)^{i} d_{\mathcal{G}}: \Omega^{(i, j, k)}(\mathcal{A} \times \mathcal{G} \times P) \rightarrow \Omega^{(i, j+1, k)}(\mathcal{A} \times \mathcal{G} \times P) \\
& \hat{d}_{P}:=(-1)^{i+j} d_{P}: \Omega^{(i, j, k)}(\mathcal{A} \times \mathcal{G} \times P) \rightarrow \Omega^{(i, j, k+1)}(\mathcal{A} \times \mathcal{G} \times P) .
\end{aligned}
$$

We consider the pullback connection $\tilde{\gamma}:=\left(\mathcal{R}^{\mathcal{A}} \times i d_{P}\right)^{*} \gamma$, where $\gamma$ is the connection of (2.7). The components of $\tilde{\gamma}$ with respect to the product structure of $\Omega(\mathcal{A} \times \mathcal{G} \times P)$ are given by

$$
\begin{aligned}
& \tilde{\gamma}_{(A, v, p)}^{(1,0,0)}\left(\tau_{A}\right)=\left(\left(\left(\mathcal{R}_{v}^{\mathcal{A}}\right)^{*} \alpha\right)_{A}\right)\left(\tau_{A}\right)(p)=\operatorname{ad}\left(\hat{v}(p)^{-1}\right)\left(\alpha_{A}\left(\tau_{A}\right)\right)(p) \\
& \tilde{\gamma}_{(A, v, p)}^{(0,1,0)}\left(\mathcal{Y}_{v}\right)=\left(\Theta_{v}\left(\mathcal{Y}_{v}\right)\right)(p) \\
& \tilde{\gamma}_{(A, v, p)}^{(0,0,1)}\left(X_{p}\right)=\left(v^{*} A\right)_{p}\left(X_{p}\right)
\end{aligned}
$$


where $\tau_{A} \in T_{A} \mathcal{A}, \mathcal{Y}_{v} \in T_{v} \mathcal{G}$ and $X_{p} \in T_{p} P$. It is not difficult to calculate the curvature $\tilde{\Omega}$ of $\tilde{\gamma}$. In fact,

$$
\begin{aligned}
\tilde{\Omega}= & d_{\mathcal{A} \times \mathcal{G} \times P} \tilde{\gamma}+\frac{1}{2}[\tilde{\gamma}, \tilde{\gamma}] \\
= & \left(\mathcal{R}_{v}^{\mathcal{A}}\right)^{*} \mathcal{F}+d_{\mathcal{G}} \Theta+\frac{1}{2}[\Theta, \Theta]+v^{*} F_{A}+d_{\mathcal{A}}\left(v^{*} A\right)-d_{v^{*} A}\left(\mathcal{R}_{v}^{\mathcal{A}}\right)^{*} \alpha \\
& +d_{\mathcal{G}}\left(\left(\mathcal{R}_{v}^{\mathcal{A}}\right)^{*} \alpha\right)-\left[\left(\mathcal{R}_{v}^{\mathcal{A}}\right)^{*} \alpha, \Theta\right]+d_{\mathcal{G}}\left(v^{*} A\right)-d_{v^{*} A} \Theta .
\end{aligned}
$$

Let $\tau_{A} \in T_{A} \mathcal{A}$ be a fixed tangent vector then $v \mapsto\left(\mathcal{R}_{v}^{\mathcal{A}} * \alpha\right)_{A}\left(\tau_{A}\right)$ can be viewed as a $\operatorname{Lie} \mathcal{G}$-valued function on $\mathcal{G}$. The vector $\mathcal{Y}_{v}=\mathcal{Y}_{\Theta_{v}\left(\mathcal{Y}_{v}\right)}^{\text {left }}(v) \in T_{v} \mathcal{G}$ generates the flow $F l_{t}^{\Theta_{v}\left(\mathcal{Y}_{v}\right)}(v)=\hat{v} \cdot \operatorname{expt} \Theta_{v}\left(\mathcal{Y}_{v}\right)$. So we find

$$
\begin{aligned}
\left(d_{\mathcal{G}}\left(\left(\left(\mathcal{R}^{\mathcal{A}}\right)^{*} \alpha\right)_{A}\left(\tau_{A}\right)\right)_{v}\left(\mathcal{Y}_{v}\right)(p)\right. & =\left.\frac{d}{d t}\right|_{t=0} \operatorname{ad}\left(\exp \left(-t \Theta_{v}\left(\mathcal{Y}_{v}\right)(p)\right)\right) \operatorname{ad}\left(\hat{v}^{-1}(p)\right)\left(\alpha_{A}\left(\tau_{A}\right)\right)(p) \\
& =\left[\left(\left(\mathcal{R}_{v}^{\mathcal{A}}\right)^{*} \alpha\right)_{A}\left(\tau_{A}\right), \Theta_{v}\left(\mathcal{Y}_{v}\right)\right](p) .
\end{aligned}
$$

Inserting (2.14) and (4.5) into (4.4) leads to

4.6 Proposition. The components of the curvature $\tilde{\Omega}$ of the connection $\tilde{\gamma}$ are given by

$$
\begin{aligned}
& \tilde{\Omega}_{(A, v, p)}^{(2,0,0)}\left(\tau_{A}^{1}, \tau_{A}^{2}\right)=\left(\left(\left(\mathcal{R}_{v}^{\mathcal{A}}\right)^{*} \mathcal{F}\right)_{A}\left(\tau_{A}^{1}, \tau_{A}^{2}\right)\right)(p)=\operatorname{ad}\left(\hat{v}(p)^{-1}\right)\left(\mathcal{F}_{A}\left(\tau_{A}^{1}, \tau_{A}^{2}\right)\right)(p) \\
& \tilde{\Omega}_{(A, v, p)}^{(0,0,2)}\left(X_{p}^{1}, X_{p}^{2}\right)=\left(v^{*} F_{A}\right)_{p}\left(X_{p}^{1}, X_{p}^{2}\right) \\
& \tilde{\Omega}_{(A, v, p)}^{(1,0,1)}\left(\tau_{A}, X_{p}\right)=\left(v^{*} \tau_{A}^{h}\right)_{p}\left(X_{p}\right) \\
& \tilde{\Omega}^{(0,2,0)}=\tilde{\Omega}^{(1,1,0)}=\tilde{\Omega}^{(0,1,1)}=0 .
\end{aligned}
$$

Let us define a free $\mathcal{G}$ action on $\mathcal{A} \times \mathcal{G} \times P$ by

$$
\tilde{\mathcal{R}}_{u}(A, v, p):=\left(\mathcal{R}_{u}^{\mathcal{A}} \times A d\left(u^{-1}\right) \times u^{-1}\right)(A, v, p)=\left(u^{*} A, u^{-1} v u, u^{-1}(p)\right),
$$

then it is easily verified that $\left(\mathcal{R}^{\mathcal{A}} \times i d_{P}\right) \circ \tilde{\mathcal{R}}_{u}=\mathcal{R}_{u} \circ\left(\mathcal{R}^{\mathcal{A}} \times i d_{P}\right)$ holds. Since $\gamma$ is $\mathcal{G}$ invariant we find $\tilde{\mathcal{R}}_{u} \tilde{\gamma}=\tilde{\gamma}$. The quotient $(\mathcal{A} \times \mathcal{G} \times P) / \mathcal{G}$ admits a canonical free $G$ action. The induced $\mathcal{G}$-action on $\mathcal{A} \times \mathcal{G} \times M$ is given by $(A, v, x) \mapsto\left(u^{*} A, u^{-1} v u, x\right)$. Let $A d \mathcal{A}=\mathcal{A} \times{ }_{\mathcal{G}} \mathcal{G}$ denote the adjoint bundle, associated to the principal bundle $\mathcal{A}$ via the adjoint action of $\mathcal{G}$ onto itself. Then one can prove $(\mathcal{A} \times \mathcal{G} \times M) / \mathcal{G} \cong A d \mathcal{A} \times M$ and thus $(\mathcal{A} \times \mathcal{G} \times P) / \mathcal{G} \rightarrow A d \mathcal{A} \times M$ becomes a principal $G$-bundle, denoted by $\hat{\mathcal{P}}$. In summary there exists the following commutative diagram of principal bundles

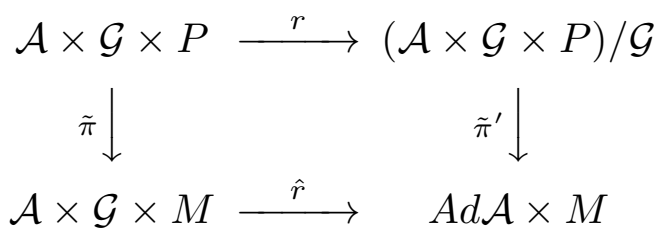

The vertical subspaces $V_{(A, v, p)}^{r}(\mathcal{A} \times \mathcal{G} \times P)$ of the principal bundle $\mathcal{A} \times \mathcal{G} \times P \rightarrow$ $(\mathcal{A} \times \mathcal{G} \times P) / \mathcal{G}$ are given by

$$
V_{(A, v, p)}^{r}(\mathcal{A} \times \mathcal{G} \times P)=\left\{\left(d_{A} \xi, \mathcal{Y}_{\xi}^{\text {left }}(v)-\mathcal{Y}_{\xi}^{\text {right }}(v),-Z_{\xi}(p)\right) \mid \xi \in \Omega^{0}(M, a d P)\right\},
$$

where $\mathcal{Y}_{\xi}^{\text {right }}$ denotes the right invariant vector field on $\mathcal{G}$ induced by $\xi$. It is easy to show that $\left.\tilde{\gamma}\right|_{V^{r}(\mathcal{A} \times \mathcal{G} \times P)}=0$ and so we end up with 
4.10 Proposition. The connection $\tilde{\gamma}=\left(\mathcal{R}^{\mathcal{A}} \times i d_{P}\right)^{*} \gamma$ descends to a well defined connection on the $G$-bundle $(\mathcal{A} \times \mathcal{G} \times P) / \mathcal{G} \rightarrow A d \mathcal{A} \times M$.

It is clear that $\hat{r}^{*} \hat{\mathcal{P}} \cong \mathcal{P}$. So $\mathcal{P}$ with connection $\tilde{\gamma}$ can serve as corresponding generalization of $\mathcal{Q}$ with universal connection $\gamma$.

Let $\tilde{i}_{A}: \mathcal{G} \times \mathcal{G} \times P \hookrightarrow \mathcal{A} \times \mathcal{G} \times P,(u, v, p) \mapsto\left(u^{*} A, v, p\right)$ be the embedding of the gauge orbit through $A \in \mathcal{A}$. The components of the restriction of $\tilde{\gamma}$ are given by

$$
\begin{aligned}
& \left(\tilde{i}_{A}^{*} \tilde{\gamma}\right)_{(u, v, p)}^{(1,0,0)}\left(\mathcal{Y}_{u}\right)=\operatorname{ad}\left(\hat{v}(p)^{-1}\right) \Theta_{u}\left(\mathcal{Y}_{u}\right)(p) \\
& \left(\tilde{i}_{A}^{*} \tilde{\gamma}\right)_{(u, v, p)}^{(0,1,0)}\left(\mathcal{Y}_{v}\right)=\Theta_{v}\left(\mathcal{Y}_{v}\right)(p) \\
& \left(\tilde{i}_{A}^{*} \tilde{\gamma}\right)_{(u, v, p)}^{(0,0,1)}\left(X_{p}\right)=\left((u v)^{*} A\right)_{p}\left(X_{p}\right) .
\end{aligned}
$$

Since $\tilde{\Omega}$ is horizontal with respect to the principal fibration $\mathcal{A} \rightarrow \mathcal{M}$, a generalization of the Russian formula holds, namely

$$
\left(\tilde{i}_{A}^{*} \tilde{\Omega}\right)_{(u, v, p)}\left(\left(\mathcal{Y}_{u}^{1}, \mathcal{Y}_{v}^{\prime 1}, X_{p}^{1}\right),\left(\mathcal{Y}_{u}^{2}, \mathcal{Y}_{v}^{\prime 2}, X_{p}^{2}\right)\right)=\left((u v)^{*} F_{A}\right)_{p}\left(X_{p}^{1}, X_{p}^{2}\right) .
$$

Now we define $\tilde{A}:=\tilde{i}_{A}^{*} \tilde{\gamma}^{(0,0,1)}, \bar{\Theta}:=\tilde{i}_{A}^{*} \tilde{\gamma}^{(1,0,0)}$ and $\tilde{F}_{A}:=\tilde{i}_{A}^{*} \tilde{\Omega}$. Because of (4.12) the components of $\tilde{i}_{A}^{*} \tilde{\gamma}$ fulfill the relations

$$
\begin{array}{ll}
d_{\mathcal{G}}^{(1)} \tilde{A}-d_{\tilde{A}} \bar{\Theta}=0, & d_{\mathcal{G}}^{(2)} \tilde{A}-d_{\tilde{A}} \Theta=0 \\
d_{\mathcal{G}}^{(1)} \bar{\Theta}+\frac{1}{2}[\bar{\Theta}, \bar{\Theta}]=0, & d_{\mathcal{G}}^{(2)} \Theta+\frac{1}{2}[\Theta, \Theta]=0 \\
d_{\mathcal{G}}^{(2)} \bar{\Theta}+[\bar{\Theta}, \Theta]=0, & d_{\mathcal{G}}^{(1)} \Theta=0,
\end{array}
$$

where $d_{\mathcal{G}}^{(1)}$ and $d_{\mathcal{G}}^{(2)}$ denote the exterior derivatives with respect to the first and second factor in the product $\mathcal{G} \times \mathcal{G} \times P$. Furthermore the Bianchi identity gives

$$
d_{\mathcal{G}}^{(1)} \tilde{F}_{A}+\left[\bar{\Theta}, \tilde{F}_{A}\right]=0 \quad d_{\mathcal{G}}^{(2)} \tilde{F}_{A}+\left[\Theta, \tilde{F}_{A}\right]=0
$$

In comparison with [Ts, Z] we can identify $d_{\mathcal{G}}^{(1)}$ with the anti-BRS operator and $d_{\mathcal{G}}^{(2)}$ with the BRS operator. Moreover $\Theta$ is identified with the ghost and $\bar{\Theta}$ plays the role of the anti-ghost field. Thus we have shown that the BRS/anti-BRS multiplet can be geometrically realized on the $G$-bundle $\mathcal{G} \times \mathcal{G} \times P \rightarrow \mathcal{G} \times \mathcal{G} \times M$.

Let $Q \in I^{m}(G)$ and define the $2 m$ form $Q(\tilde{\Omega})$. The transgression formula $Q(\tilde{\Omega})=$ $d_{\mathcal{A} \times \mathcal{G} \times P} T Q(\tilde{\gamma})$ gives rise to general descent equations. However, by restricting to the gauge orbit through $A \in \mathcal{A}$ this system can be separated into a set, which corresponds to the cohomology of $d_{\mathcal{G}}^{(1)}$ and $d_{\mathcal{G}}^{(2)}$ respectively

$$
\begin{aligned}
Q\left(\tilde{i}_{A}^{*} \tilde{\Omega}\right)^{(0,0,2 m)} & =d_{P} T Q\left(\tilde{i}_{A}^{*} \tilde{\gamma}\right)^{(0,0,2 m-1)} & & \\
0 & =d_{\mathcal{G}}^{(2)} T Q\left(\tilde{i}_{A}^{*} \tilde{\gamma}\right)^{(0, k-1,2 m-k)}+\hat{d}_{P} T Q\left(\tilde{i}_{A}^{*} \tilde{\gamma}\right)^{(0, k, 2 m-1-k)} & & 1 \leqq k \leqq 2 m \\
0 & =d_{\mathcal{G}}^{(1)} T Q\left(\tilde{i}_{A}^{*} \tilde{\gamma}\right)^{(k-1,0,2 m-k)}+\hat{d}_{P} T Q\left(\tilde{i}_{A}^{*} \tilde{\gamma}\right)^{(k, 0,2 m-1-k)} & & 1 \leqq k \leqq 2 m
\end{aligned}
$$


There is also a mixed set of equations for $1 \leqq k \leqq 2 m$

$$
\begin{aligned}
& 0=d_{\mathcal{G}}^{(1)} T Q\left(\tilde{i}_{A}^{*} \tilde{\gamma}\right)^{(0, k-1,2 m-k)}+\hat{d}_{\mathcal{G}}^{(2)} T Q\left(\tilde{i}_{A}^{*} \tilde{\gamma}\right)^{(1, k-2,2 m-k)}+\hat{d}_{P} T Q\left(\tilde{i}_{A}^{*} \tilde{\gamma}\right)^{(1, k-1,2 m-k-1)} \\
& 0=d_{\mathcal{G}}^{(1)} T Q\left(\tilde{i}_{A}^{*} \tilde{\gamma}\right)^{(k-2,1,2 m-k)}+\hat{d}_{\mathcal{G}}^{(2)} T Q\left(\tilde{i}_{A}^{*} \tilde{\gamma}\right)^{(k-1,0,2 m-k)}+\hat{d}_{P} T Q\left(\tilde{i}_{A}^{*} \tilde{\gamma}\right)^{(k-1,1,2 m-k-1)} .
\end{aligned}
$$

Since the components of $\tilde{\Omega}_{t}=t \tilde{\Omega}+\frac{t^{2}-t}{2}[\tilde{\gamma}, \tilde{\gamma}] \mathrm{read}$

$$
\begin{aligned}
& \tilde{\Omega}_{t} \stackrel{(2,0,0)}{(A, v, p)}\left(\tau_{A}^{1}, \tau_{A}^{2}\right)=\left(\left(\mathcal{R}_{v}^{\mathcal{A}}\right)^{*} \mathcal{F}_{t}\right)_{A}\left(\tau_{A}^{1}, \tau_{A}^{2}\right)(p) \\
& \tilde{\Omega}_{t} \stackrel{(0,2,0)}{(A, v, p)}\left(\mathcal{Y}_{v}^{1}, \mathcal{Y}_{v}^{2}\right)=\left(\frac{t^{2}-t}{2}\left[\Theta_{v}, \Theta_{v}\right]\right)\left(\mathcal{Y}_{v}^{1}, \mathcal{Y}_{v}^{2}\right)(p) \\
& \tilde{\Omega}_{t} \stackrel{(0,0,2)}{(A, v, p)}\left(X_{p}^{1}, X_{p}^{2}\right)=\left(v^{*}\left(F_{A}\right)_{t}\right)_{p}\left(X_{p}^{1}, X_{p}^{2}\right) \\
& \tilde{\Omega}_{t}{ }_{(A, 1,0),}\left(\tau_{A}, \mathcal{Y}_{v}\right)=\left(t^{2}-t\right)\left[\left(\left(\mathcal{R}_{v}^{\mathcal{A}}\right)^{*} \alpha\right)_{A}, \Theta_{v}\right]\left(\tau_{A}, \mathcal{Y}_{v}\right)(p) \\
& \tilde{\Omega}_{t} \stackrel{(0,1,1)}{(A, v, p)}\left(\mathcal{Y}_{v}, X_{p}\right)=\left(t^{2}-t\right)\left[\Theta_{v}, v^{*} A\right]\left(\mathcal{Y}_{v}, X_{p}\right) \\
& \tilde{\Omega}_{t}{ }_{(A, 0,0, p)}^{(1,0,1)}\left(\tau_{A}, X_{p}\right)=\left(t \tilde{\Omega}_{(A, v, p)}^{(1,0,1)}+\left(t^{2}-t\right)\left[\left(\left(\mathcal{R}_{v}^{\mathcal{A}}\right)^{*} \alpha\right)_{A}, u^{*} A\right]\right)\left(\tau_{A}, X_{p}\right),
\end{aligned}
$$

we obtain, in view of $(2.17)$

$$
T Q\left(\tilde{i}_{A}^{*} \tilde{\gamma}\right)_{\left(u, i d_{\mathcal{G}}, p\right)}^{(k, 0,2 m-k-1)}=T Q\left(\bar{i}_{A}^{*} \gamma\right)_{(u, p)}^{(k, 2 m-k-1)} .
$$

So we have recovered the usual expression for the consistent anomalies.

In order to formulate covariant descent equations in our present setup, we define the following connection

$$
\eta_{(A, v, p)}\left(\tau_{A}, \mathcal{Y}_{v}, X_{p}\right)=\left(v^{*} A\right)_{p}\left(X_{p}\right)+\left(\Theta_{v}\left(\mathcal{Y}_{v}\right)\right)(p)
$$

on the bundle $\mathcal{A} \times \mathcal{G} \times P \rightarrow \mathcal{A} \times \mathcal{G} \times M$. However, $\eta$ is not the pullback of $\gamma_{0}$. It is $\mathcal{G}$ invariant since

$$
\begin{aligned}
\left(\tilde{\mathcal{R}}_{u}^{*} \eta\right)_{(A, v, p)} & =\eta_{\left(u^{*} A, A d\left(u^{-1}\right) v, u^{-1}(p)\right)}\left(u^{*} \tau_{A}, T_{v} A d\left(u^{-1}\right) \mathcal{Y}_{v}, T_{p} u^{-1} X_{p}\right) \\
& =\left((v u)^{*} A\right)_{u^{-1}(p)}\left(T_{p} u^{-1} X_{p}\right)+\operatorname{ad}\left(\hat{u}\left(u^{-1}(p)\right)\right)\left(\Theta_{v}\left(\mathcal{Y}_{v}\right)\right)\left(u^{-1}(p)\right) \\
& =\eta_{(A, v, p)}\left(\tau_{A}, \mathcal{Y}_{v}, X_{p}\right) .
\end{aligned}
$$

The curvature $\Omega_{\eta}$ of $\eta$ is given by

$$
\begin{aligned}
\Omega_{\eta} & =d_{\mathcal{A} \times \mathcal{G} \times P} \eta+\frac{1}{2}[\eta, \eta] \\
& =v^{*} F_{A}+\hat{d}_{\mathcal{G}} v^{*} A-d_{v^{*} A} \Theta_{v}+d_{\mathcal{A}} v^{*} A \\
& =v^{*} F_{A}+d_{\mathcal{A}} v^{*} A,
\end{aligned}
$$

where the BRS relations (2.14) have been used. In summary, the components of $\Omega_{\eta}$ read

$$
\begin{aligned}
& \Omega_{\eta}^{(2,0,0)}=\Omega_{\eta}^{(0,2,0)}=\Omega_{\eta}^{(1,1,0)}=\Omega_{\eta}^{(0,1,1)}=0 \\
& \Omega_{\eta}{ }_{(A, 0, v) p)}^{(0,2)}\left(X_{p}^{1}, X_{p}^{2}\right)=\left(v^{*} F_{A}\right)_{p}\left(X_{p}^{1}, X_{p}^{2}\right) \\
& \Omega_{\eta}^{(1,0,1)}\left(\tau_{A}, X_{p}\right)=\left(v^{*} \tau_{A}\right)_{p}\left(X_{p}\right) .
\end{aligned}
$$


The corresponding transgression formula is $Q\left(\Omega_{\eta}\right)=d_{\mathcal{A} \times \mathcal{G} \times P} T Q(\eta)$. Using (4.13), the components of $\left(\Omega_{\eta}\right)_{t}=t \Omega_{\eta}+\frac{t^{2}-t}{2}[\eta, \eta]$ read

$$
\begin{aligned}
& \left(\Omega_{\eta}\right)_{t}{ }^{(2,0,0)}=\left(\Omega_{\eta}\right)_{t}{ }^{(1,1,0)}=0 \\
& \left(\Omega_{\eta}\right)_{t} \underset{(A, v, p)}{(0,0,2)}\left(X_{p}^{1}, X_{p}^{2}\right)=\left(v^{*}\left(F_{A}\right)_{t}\right)_{p}\left(X_{p}^{1}, X_{p}^{2}\right) \\
& \left(\Omega_{\eta}\right)_{t} \stackrel{(1,0,1)}{(A, v, p)}\left(\tau_{A}, X_{p}\right)=t d_{\mathcal{A}}\left(v^{*} A\right)\left(\tau_{A}, X_{p}\right)=t\left(v^{*} \tau_{A}\right)_{p}\left(X_{p}\right)
\end{aligned}
$$

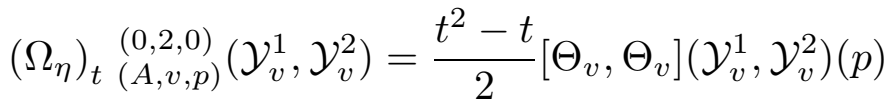

$$
\begin{aligned}
& \left(\Omega_{\eta}\right)_{t}^{\stackrel{(0,1,1)}{(A, v, p)}}\left(\mathcal{Y}_{v}, X_{p}\right)=\left(t^{2}-t\right)\left[\Theta_{v}, v^{*} A\right]\left(\mathcal{Y}_{v}, X_{p}\right) \text {. }
\end{aligned}
$$

Hence we find the important relation

$$
T Q\left(\tilde{i}_{A}^{*} \eta\right)_{\left(u, i d_{\mathcal{G}, p)}\right.}^{(j, 0,2 m-j-1)}=T Q\left(\bar{i}_{A}^{*} \gamma_{0}\right)_{(u, p)}^{(j, 2 m-j-1)}
$$

Moreover (4.6) and (4.22) imply

$$
\begin{aligned}
Q(\tilde{\Omega})^{(j, k-j, 2 m-k)} & =0, & & j \neq k \\
Q\left(\Omega_{\eta}\right)^{(j, k-j, 2 m-k)} & =0, & & j \neq k .
\end{aligned}
$$

The transgression formula $Q(\tilde{\Omega})-Q\left(\Omega_{\eta}\right)=d_{\mathcal{A} \times \mathcal{G} \times P} T Q(\tilde{\gamma}, \eta)$ leads to the descent equations

$$
\begin{aligned}
\left(Q(\tilde{\Omega})-Q\left(\Omega_{\eta}\right)\right)^{(j, k-j, 2 m-k)}= & d_{\mathcal{A}} T Q(\tilde{\gamma}, \eta)^{(j-1, k-j, 2 m-k)}+\hat{d}_{\mathcal{G}} T Q(\tilde{\gamma}, \eta)^{(j, k-j-1,2 m-k)} \\
& +\hat{d}_{P} T Q(\tilde{\gamma}, \eta)^{(j, k-j, 2 m-k-1)}
\end{aligned}
$$

where $0 \leq j, k \leq 2 m$. Furthermore (4.20) implies $\left(\mathcal{R}_{u}^{\mathcal{A}} \times i d_{\mathcal{G}} \times i d_{P}\right)^{*} T Q(\tilde{\gamma}, \eta)=$ $T Q(\tilde{\gamma}, \eta)$. The components of the curvature $\tilde{\Omega}_{t}^{\prime}$ corresponding to the connection $t \tilde{\gamma}+(1-t) \eta$ are

$$
\begin{aligned}
& \tilde{\Omega}_{t}^{\prime(0,2,0)}=\tilde{\Omega}_{t}^{\prime(0,1,1)}=\tilde{\Omega}_{t}^{\prime(1,1,0)}=0 \\
& \tilde{\Omega}_{t}^{\prime(A, 0,0)}\left(\tau_{A}^{1}, \tau_{A}^{2}\right)=\left(\left(\mathcal{R}_{v}^{\mathcal{A}}\right)^{*} \mathcal{F}_{t}\right)_{A}\left(\tau_{A}^{1}, \tau_{A}^{2}\right)(p) \\
& \tilde{\Omega}_{t}^{\prime(0,0,2)}\left(X_{p}^{1}, X_{p}^{2}\right)=\left(v^{*} F_{A}\right)_{p}\left(X_{p}^{1}, X_{p}^{2}\right) \\
& \tilde{\Omega}_{t(A, v, p)}^{(1,0,1)}\left(\tau_{A}, X_{p}\right)=\left(d_{\mathcal{A}}\left(v^{*} A\right)-t d_{v^{*} A}\left(\left(\mathcal{R}_{v}^{\mathcal{A}}\right)^{*} \alpha_{A}\right)\right)\left(\tau_{A}, X_{p}\right),
\end{aligned}
$$

where we have used the BRS/anti-BRS relations (4.13). It is obvious from (3.8) and (4.27) that

$$
T Q\left(\tilde{i}_{A}^{*} \tilde{\gamma}, \tilde{i}_{A}^{*} \eta\right)_{\left(u, i d_{\mathcal{G}}, p\right)}^{(j, 0,2 m-j-1)}=T Q\left(\bar{i}_{A}^{*} \gamma, \bar{i}_{A}^{*} \gamma_{0}\right)_{(u, p)}^{(j, 2 m-j-1)}
$$

holds. Generally, in consequence of (4.27) one finds

$$
T Q(\tilde{\gamma}, \eta)^{(j, k-j, 2 m-k-1)}=0 \quad j \neq k
$$


Choose $k=j+1$, then the descent equation (4.26) reduces to

$$
d_{\mathcal{G}} T Q(\tilde{\gamma}, \eta)^{(j, 0,2 m-j-1)}=0
$$

and so one ends up with

$$
d_{\mathcal{G}}^{(2)} T Q\left(\tilde{i}_{A}^{*} \tilde{\gamma}, \tilde{i}_{A}^{*} \eta\right)^{(j, 0,2 m-j-1)}=0,
$$

which in $[\mathrm{AAG}]$ is called the strong covariance condition for the non-integrated covariant anomalies. However, this result could have been also obtained by a direct computation using the BRS/anti-BRS transformations (4.13), (4.14) and the property of $Q$ being ad-invariant. Thus we have shown that the solutions of the covariant descent equations automatically satisfy the covariance condition.

In view of

$$
T Q(\tilde{\gamma}, \eta)=T Q(\tilde{\gamma})-T Q(\eta)+d_{\mathcal{A} \times \mathcal{G} \times P} S_{Q}(\tilde{\gamma}, \eta),
$$

and following the procedure of Sec. 2, the counterterm relating consistent and covariant anomalies may be defined by

$$
\begin{aligned}
\lambda(\tilde{\gamma}, \eta)^{(j, 0,2 m-j-1)}:= & T Q(\eta)^{(j, 0,2 m-j-1)}-d_{\mathcal{A}} S_{Q}(\tilde{\gamma}, \eta)^{(j-1,0,2 m-j-1)} \\
& +(-1)^{j+1} d_{P} S_{Q}(\tilde{\gamma}, \eta)^{(j, 0,2 m-j-2)} .
\end{aligned}
$$

Note that $S_{Q}(\tilde{\gamma}, \eta)^{(0, k, 2 m-k-2)}=0$, since $(\tilde{\gamma}-\eta) \in \Omega^{(1,0,0)}(\mathcal{A} \times \mathcal{G} \times P, \mathfrak{g})$. For sake of completeness we want to write the covariance condition (4.31) in terms of the consistent anomaly and the corresponding counterterm. Let us assume, for simplicity, that $P=M \times G$ is trivial and let $\sigma$ denote a global section of $\mathcal{G} \times \mathcal{G} \times P \rightarrow \mathcal{G} \times \mathcal{G} \times M$. Using (4.31) and (4.33), we find

$$
\begin{gathered}
0=d_{\mathcal{G}}^{(2)} \int_{N} \sigma^{*}\left[T Q\left(\tilde{i}_{A}^{*} \tilde{\gamma}\right)^{(j, 0,2 m-j-1)}-\left(T Q\left(\tilde{i}_{A}^{*} \eta\right)^{(j, 0,2 m-j-1)}\right.\right. \\
\left.\left.-d_{\mathcal{G}}^{(1)} S_{Q}\left(\tilde{i}_{A}^{*} \tilde{\gamma}, \tilde{i}_{A}^{*} \eta\right)^{(j-1,0,2 m-j-1}\right)\right]
\end{gathered}
$$

where $N$ is a $(2 m-j-1)$ dimensional submanifold of $M$. For $j=1$, (4.34) reduces to the well known covariance condition for the integrated covariant current anomaly

$$
d_{\mathcal{G}}^{(2)} \int_{M} \sigma^{*}\left[T Q\left(\tilde{i}_{A}^{*} \tilde{\gamma}\right)^{(1,0,2 m-2)}-T Q\left(\tilde{i}_{A}^{*} \eta\right)^{(1,0,2 m-2)}\right]=0 .
$$

It has been argued in Ref. 6 that two homotopy operators have to be introduced to generate the covariant anomalies. In our approach this assertion is nothing but the fact that the counterterm $\lambda\left(\tilde{i}_{A}^{*} \tilde{\gamma}, \tilde{i}_{A}^{*} \eta\right)^{(j, 0,2 m-j-1)}$ consists of two different types of local polynomials, namely $T Q\left(\tilde{i}_{A}^{*} \eta\right)^{(j, 0,2 m-j-1)}$ and $S_{Q}\left(\tilde{i}_{A}^{*} \tilde{\gamma}, \tilde{i}_{A}^{*} \eta\right)^{(j, 0,2 m-j-1)}$. However, if $P \rightarrow M$ is non-trivial the generalization of the background formalism discussed previously is straightforward.

Using (4.32), one finds from (4.26)

$$
\begin{aligned}
Q\left(\tilde{i}_{A}^{*} \Omega_{\eta}\right)^{(1,0,2 m-1)} & =d_{P} T Q\left(\tilde{i}_{A}^{*} \tilde{\gamma}, \tilde{i}_{A}^{*} \eta\right)^{(1,0,2 m-2)} \\
& =d_{P}\left(T Q\left(\tilde{i}_{A}^{*} \tilde{\gamma}\right)^{(1,0,2 m-2)}-T Q\left(\tilde{i}_{A}^{*} \eta\right)^{(1,0,2 m-2)}\right) .
\end{aligned}
$$


If $n=\operatorname{dim} M=2 m-2$, the left hand side of (4.36) vanishes and so the nonintegrated covariant current anomaly induces the local de-Rham cohomology class

$$
\left[T Q\left(\tilde{i}_{A}^{*} \tilde{\gamma}, \tilde{i}_{A}^{*} \eta\right)^{(1,0, n)}\right]=\left[T Q\left(\tilde{i}_{A}^{*} \tilde{\gamma}\right)^{(1,0, n)}-T Q\left(\tilde{i}_{A}^{*} \eta\right)^{(1,0, n)}\right] \in H_{d_{P}}^{(1,0,2 m-2)}(\mathcal{G} \times \mathcal{G} \times P, \mathbb{R})
$$

in complete analogy with the result obtained in the previous section.

In summary we have found two conditions

$$
\begin{aligned}
& d_{\mathcal{G}}^{(2)} T Q\left(\tilde{i}_{A}^{*} \tilde{\gamma}, \tilde{i}_{A}^{*} \eta\right)^{(1,0, n)}=0 \\
& d_{P} T Q\left(\tilde{i}_{A}^{*} \tilde{\gamma}, \tilde{i}_{A}^{*} \eta\right)^{(1,0, n)}=0
\end{aligned}
$$

for the non-integrated covariant current anomaly. These conditions are a direct consequence of the covariant descent equations which we have derived in this paper. Thus we have shown how the covariance condition for the covariant anomaly is related with its characterization in terms of local de-Rham cohomology.

\section{ACKNOWLEDGEMENT}

I would like to thank Prof. J. Wess for his kind hospitality at the LMU of Munich.

\section{REFERENCES}

[AAG] I. Abud, J-P. Ader and F. Gieres, Algebraic determination of covariant anomalies and Schwinger terms, Nucl. Phys. B339 (1990), 687.

[AS] M.F. Atiyah and I.M. Singer, Dirac operators coupled to vector potentials, Proc. Natl. Acad. Sci. 81 (1984), 2597.

[BC1] L. Bonora and P. Cotta-Ramusino, Consistent and covariant anomalies and local cohomology, Phys. Rev. D33 (1986), 3055.

[BC2] - Some remarks on BRS transformations, anomalies and the cohomology of the Lie algebra of the group of gauge transformations, Commun. Math. Phys. 87 (1983), 589.

[BZ] W.A. Bardeen and B. Zumino, Consistent and covariant anomalies in gauge and gravitational theories, Nucl. Phys. B244 (1984), 421.

[C] S.S. Chern, Complex manifolds without potential theory, Springer, New York, 1979.

[DTV] M. Dubois-Violette, M. Talon and C.M. Viallet, BRS algebras, analysis of the consistency equations in gauge theories, Commun. Math. Phys. 102 (1985), 105.

[F] L. Faddeev, Operator anomaly for the Gauss law, Phys. Lett. B145 (1984), 81.

[K1] G. Kelnhofer, On the algebraic structure of covariant anomalies and covariant Schwinger terms, preprint UWThPh-1992-22 (1992).

[K2] Determination of covariant Schwinger terms in anomalous gauge theories, Z. Phys.C 52 (1991), 89.

[M] J. Mickelsson, Chiral anomalies in even and odd dimensions, Commun. Math. Phys. 97 (1985), 361.

[MSZ] J. Manes, R. Stora and B. Zumino, Algebraic study of chiral anomalies, Commun. Math. Phys. 102 (1985), 157.

[MV] P. Mitter and C.M. Viallet, On the bundle of connections and the gauge orbit manifold in Yang-Mills theory, Commun. Math. Phys. 79 (1981), 457.

[NT] T. Nishikawa and I. Tsutsui, Consistent and covariant commutator anomalies in gauge theories, Nucl. Phys. B308 (1988), 544.

[S] R. Stora, Progress in gauge field theories (H. Lehmann, eds.), Plenum Press, New York, 1984.

[Tr] A. Tröster, Non-abelian anomalies and the Atiyah-Singer index theorem, preprint UWThPh1991- 30 (1991). 
[Ts] I. Tsutsui, Covariant anomalies in cohomology approach, Phys. Lett. B229 (1989), 51.

[WZ] J. Wess and B. Zumino, Consequences of anomalous Ward identies, Phys. Lett. B37 (1971), 95.

[Z] Y.Z. Zhang, Covariant anomaly and cohomology in connection space, Phys. Lett. B219 (1989), 439.

[Zu] B. Zumino, Relativity, groups and topology II (B.S. De Witt and R. Stora, eds.), NorthHolland, Amsterdam, 1984.

[ZWZ] I. Zumino, Y.S. Wu and A. Zee, Chiral anomalies, higher dimensions, and differential geometry, Nucl. Phys. B239 (1984), 477. 